\title{
Conocimientos y prácticas sobre parásitos intestinales en una comunidad del pueblo indígena Zenú, Colombia, 2019
}

\section{Knowledge and practices about intestinal parasites in a community of the zenú indigenous people, Colombia, 2019}

\author{
Jaime Gómez Díaz' Francisco Ramírez Mejía ${ }^{2}$ Concepción Amador Ahumada ${ }^{3}$ (ina Valencia Jiménez ${ }^{4}$
}

Recibido para publicación: Mayo 312020 - Aprobado para publicación: Diciembre 022020

\begin{abstract}
RESUMEN
Introducción. Se desconocen los conocimientos y prácticas que tiene el Pueblo Indígena Zenú frente a los parásitos intestinales, lo que impide la planificación y atención integral por parte de las autoridades de salud en la población correspondiente. Objetivo. Describir conocimientos y prácticas relacionadas a parásitos intestinales de la comunidad indígena Zenú ubicada en la vereda Berlín - San Andrés de Sotavento. Materiales y métodos. Investigación cualitativa, etnográfica, que permite identificar la perspectiva cultural del pueblo indígena Zenú; comunidad residente en la vereda de Berlín. El instrumento empleado fue la entrevista semiestructurada; diseñada para recolectar la información sobre los conocimientos y prácticas relacionadas con las parasitosis intestinales, en atención a cinco categorías específicas; percepciones, ciclo de vida, riesgos percibidos, tratamiento y prevención. El registro de la información fue grabado y escrito, previo consentimiento de los participantes. La entrevista se aplicó a dos grupos focales; uno conformados por 12 niños, y otro conformado por 12 adultos. El muestreo empleado fue por conveniencia. Resultados. Se identificó la mezcla de conocimientos y prácticas de autocuidado provenientes de la biomedicina y el saber tradicional. Conclusiones. Mujeres adultas y niños identifican a los parásitos intestinales como causantes de padecimientos, e identifican -desde la óptica biomédica- algunos síntomas y formas de prevención relacionadas a esta patología, mezclada en ocasiones con el conocimiento tradicional. Se identificó el desarrollo de acciones de autocuidado basados en el conocimiento de la medicina ancestral de los zenúes. Los sujetos de estudio ignoran los riesgos que implica el consumo de agua contaminada o sin tratar.
\end{abstract}

Palabras clave: parásitos intestinales, población indígena, conocimientos, prácticas.

\begin{abstract}
Background. The knowledge and practices of the Zenú Indigenous People about intestinal parasites are unknown, which prevents planning and comprehensive care by the health authorities in the corresponding population. Objective. Describe knowledge and practices related to intestinal parasites of the Zenú indigenous community located in the Berlin - San Andrés de Sotavento neighborhood. Materials and methods. Qualitative, ethnographic research that allows identifying the cultural perspective of people was conducted. The instrument used was the semi-structured interview; designed to collect information on knowledge and practices related to intestinal parasites, according to five specific categories; perceptions, life cycle, perceived risks, treatment and prevention. The information record was documented, with the prior consent of the participants. The interview was applied to two focus groups; one made up of 12 children, and the other made up of 12 adults. The sampling used was for convenience. Results. The mix of knowledge and self-care practices from biomedicine and traditional knowledge was identified. Conclusions. Adult women and children identify intestinal parasites as causing disease, and identify - from a biomedical perspective - some symptoms and forms of prevention related to this pathology, sometimes mixed with traditional knowledge. The development of selfcare actions based on the knowledge of the ancient medicine of the Zenúes was identified. Study subjects ignore the risks of consuming contaminated or untreated water.
\end{abstract}

Keywords: intestinal parasites, indigenous population, knowledge, practices.

Citación (Vancouver)

Gómez J, Ramínez F, Amador C, Valencia N. Conocimientos y prácticas sobre parásitos intestinales en una comunidad del pueblo indígena Zenú, Colombia, 2019. Rev Avances en Salud; 2020. (4)2:10-20. doi: 10.21897/25394622.2342

${ }^{1}$ Biólogo, MSc en Salud Pública. Facultad Ciencias de la Salud, Universidad de Córdoba. Montería, Córdoba, Colombia.

Correspondencia: eduardogomezdiaz@hotmail.com.

${ }^{2}$ Médico, MSc en Salud Pública.

${ }^{3}$ Enfermera, MSc en Enfermería.

${ }^{4}$ Desarrollista familiar, MSc en educación 


\section{INTRODUCCIÓN}

Las parasitosis intestinales son infestaciones causadas por microorganismos protozoarios y/o helmintos que pueden transmitirse por la ingesta de agua o alimentos contaminados con heces, por penetración de larvas a través de la piel, de persona a persona o de animales al hombre (1). El hábitat natural de este tipo de parásitos es el tracto gastrointestinal de los hospederos, y la presentación clínica de la enfermedad puede caracterizarse por la mala absorción intestinal, inapetencia, lesiones en la mucosa intestinal, diarrea, anemia y desnutrición $(2,3)$; lo que conlleva en muchos casos a que se disminuya el desarrollo cognitivo y el crecimiento, se generen daños irreversibles en el estado de salud de las personas y se aumente la probabilidad de morir (4). En el contexto global, las parasitosis intestinales son consideradas un grupo de enfermedades con alta prevalencia, las cuales comprometen al individuo, a la familia y a la comunidad $(5,6)$. Se estima que existen cerca de dos mil millones de personas en el mundo infectadas con parásitos intestinales, de las cuales, mil millones se encuentran parasitadas con 1 o más especies de helmintos y 74 millones con al menos una especie de protozoario, lo que constituye un problema para la salud pública por el alto riesgo de transmisión y endemicidad (7-10). Problemas generalizados como la pobreza, prácticas higiénicas deficientes, carencia de infraestructura sanitaria, características socio-antropológicas de las comunidades y niveles educativos bajos, son algunos de los factores que aumentan la susceptibilidad a estas infecciones (11-14). Para los pueblos indígenas de Colombia, el problema de las parasitosis intestinales se agrava debido a que viven en zonas distantes, donde los servicios básicos de salud son mínimos y los médicos tradicionales presentan dificultades para el diagnóstico y tratamiento $(15,16)$. Adicionalmente, el conocimiento de la medicina ancestral, que es manejado por sabedores y algunos miembros de sus comunidades -a través de rezos y remedios naturales- (17), generalmente no es tenido en cuenta por parte de las entidades de salud que actualmente prestan sus servicios dentro de los territorios indígenas, lo que impide la planificación y atención integral por parte de las autoridades de salud en la población correspondiente. En este sentido, el pueblo indígena Zenú que reside en el municipio de San Andrés de Sotavento-Córdoba, no se encuentra ajeno a esta problemática; algunas de sus comunidades no cuentan con red de agua potable, alcantarillado, ni lugares adecuados para el depósito de las excretas, las tasas de analfabetismo son elevadas y los recursos económicos de sus familias son escasos (18). Así mismo, se desconoce el comportamiento humano relacionado con las enfermedades parasitarias, desde la perspectiva de la gente misma. Por tal razón, resulta imprescindible describir los conocimientos y prácticas relacionadas a parásitos intestinales en una comunidad indígena ubicada en el municipio de San Andrés de Sotavento, como un paso inicial para el diseño de programas integrales de educación en salud (19).

\section{MATERIALES Y MÉTODOS}

\section{Diseño del estudio}

Investigación cualitativa, etnográfica, que permite identificar la perspectiva cultural de las personas (visión émica). El instrumento empleado fue la entrevista semiestructurada; diseñada para recolectar la información sobre los conocimientos y prácticas relacionadas con las parasitosis intestinales, en atención a cinco categorías específicas. El registro de la información fue grabado y escrito, previo consentimiento de los participantes. El formulario de la entrevista se probó previamente con individuos residentes en la vereda Berlín. La entrevista se aplicó posteriormente a dos grupos focales; uno conformados por 12 menores de trece años, y otro conformado 
por 12 adultos. El muestreo empleado fue por conveniencia. Cuando la información recabada con la entrevista se hizo repetitiva, se dio por finalizado el proceso de recolección. Se realizó la interpretación y análisis del contenido de los resultados.

\section{Escenario de estudio}

El estudio fue realizado en la comunidad indígena Zenú residente en la vereda Berlín, ubicada en las coordenadas geográficas $9^{\circ} 03^{\prime} 51.2^{\prime \prime} \mathrm{N} 75^{\circ} 33^{\prime} 01.5^{\prime \prime} \mathrm{W}$, a 11 kilómetros de la cabecera municipal de San Andrés de Sotavento-Córdoba. Dicha vereda se encuentra enmarcada en el área rural, por tal razón, la forma de vida y las costumbres características de la comunidad corresponden a una de las más conservadas y representativas del pueblo indígena Zenú. En la vereda Berlín residen 637 personas, agrupadas en 147 familias. La principal actividad productiva de esta comunidad es la agricultura, combinada con la crianza de animales domésticos. La tipología de vivienda corresponde en su gran mayoría a domicilios con paredes en bahareque, piso de tierra y techo de palma o zinc. Según la clasificación de las zonas de vida de L. R. Holdridge, la vereda Berlín se encuentra en un área de bosque seco tropical (bs-T) (20). El pueblo Zenú se caracteriza por emplear la medicina ancestral, la cual se encuentra estrechamente ligada con el ámbito espiritual. Este conocimiento es transmitido a través de las generaciones, lo que permite que en la actualidad aún sea posible encontrar sobanderos, parteras, médicos tradicionales, rezanderos entre otros.

\section{Procesamiento y análisis de datos}

Se realizó la deconstrucción de las respuestas obtenidas en la entrevista en pequeñas unidades comparables. Se maximizaron las semejanzas entre los datos que presentaron analogías y se ordenaron de manera aproximada. Se organizó la información resultante en categorías y se utilizó un análisis de contenido basado en los conocimientos y aportes de la Antropología y de la Socioantropología, dada la naturaleza del contexto social de los sujetos del estudio.

\section{Consideraciones éticas.}

La investigación se ciñe a la resolución No. 008430 de 1993 del Ministerio de Salud de Colombia. Fue una investigación con riesgo mínimo. Se obtuvo el consentimiento y asentimiento informado de los sujetos de estudio. Se contó con la aprobación de la autoridad tradicional de la comunidad. La información se codificó para salvaguardar la confidencialidad. El proyecto fue aprobado por el Comité de Ética de la Facultad de Ciencias de la Salud de la Universidad de Córdoba.

\section{RESULTADOS}

Las cinco categorías planteadas en la entrevista con sus respectivas preguntas son las siguientes: 1. Percepciones. a) ¿Qué saben acerca de los parásitos intestinales? b) ¿Conoce usted qué forma, aspecto o color tienen los parásitos intestinales? 2. Ciclo de vida. c) ¿Cómo entran los parásitos intestinales al cuerpo de las personas? d) ¿Qué sucede con los parásitos cuando entran al cuerpo de las personas? e) ¿Por dónde salen los parásitos del cuerpo de las personas? f) ¿Qué sucede con los parásitos cuando salen del cuerpo? 3. Riesgos percibidos. g) ¿Las personas se pueden infectar con los mismos parásitos que afectan a los animales? 4. Tratamiento. h) ¿Qué tratamiento debe recibir una persona que tiene parásitos intestinales? 5. Prevención. i) ¿Cómo se puede prevenir la infección por parásitos intestinales?

\section{Percepciones}

Ante la pregunta ¿Qué saben acerca de las parasitosis intestinales?, los niños evidenciaron que reconocen algunos síntomas que presentan personas que se encuentran infestadas con este tipo de parásitos:

- N1: "Las personas que tienen parásitos les da dolor de barriga, le suenan los dientes cuando están durmiendo y pasan con dolores de barriga". 
- N2: "Nosotros nos ponemos flacos".

- N3: "Nos da anemia".

Por otra parte, algunos padres de familia -ante la misma pregunta- identifican manifestaciones clínicas que presenta una persona infectada con parásitos intestinales, y para ampliar al respecto describen cómo examinan físicamente a un niño cuando se sospecha que tiene esta enfermedad. En el siguiente aparte, una madre detalla cómo ausculta el ano de su hijo, y describe de forma puntual cómo realizar la búsqueda de parásitos intestinales en esta parte del cuerpo. Cabe resaltar que el término "tantino" es usado por algunos miembros de la comunidad para referirse al ano o a los intestinos.

- A1: "Registrarlos de noche (...) me busco un foco, me voy de noche y les alzo...abajito, le alzo las tapitas de las nalguitas (...) y les registro el tantino y eso se le ve el parásito ¿qué hago yo?, con un trapito mojao' se los saco".

Cuando se les preguntó sobre la forma, aspecto o color de los parásitos intestinales, los niños coinciden en que estos tienen forma alargada, haciendo una descripción general de alguna especie de helminto. Sin embargo, ninguno de los niños realiza una representación verbal de los protozoarios:

- N4: "Cuando uno se toma un purgante a veces pa' botar los parásitos me ha tocado de verlos, que tienen una cabecita negra, lisito delgatido, ya vieron como una culebrita".

- N1: "Son animales chiquiticos; unos gusanitos blancos delgaditos. Pueden ser grandes, chiquitos".

Así mismo, padres de familia describen la forma de los parásitos intestinales haciendo analogía a las lombrices; delgadas y alargadas, algunas con presencia de estructuras anatómicas descritas como "conchas". A continuación, algunas respuestas de los adultos referentes al tema:

- A2: "Hay unos grandes que tienen como $50 \mathrm{~cm}$, porque yo he visto los parásitos cuando purgaba los hijos míos, hay uno que tenían como $20 \mathrm{~cm}$. Yo me acuerdo que yo purgué uno que tenía un año, le di... sino botó 500 lombrices y parásitos no botó nada. Eran largos. Dicen que hay unos que echan una conchita, tienen una cabecita negra".

- A5: "Como una lombricita, de color los he visto blanco, amarillos, chiquiticos, no tan grandes".

Cabe resaltar que son las mujeres -en su mayoría- quienes dieron respuesta a esta pregunta, mientras los hombres por lo general manifestaron no tener conocimiento ante el tema.

\section{Ciclo de vida}

Al indagar sobre las vías que los parásitos intestinales emplean para entrar al cuerpo humano, se identifica de manera implícita el tipo de conductas preventivas que toman los individuos. A continuación, algunas de las respuestas obtenidas ante la pregunta ¿Cómo entran los parásitos intestinales al cuerpo de las personas?

- N3: "Cuando los niños tienen las uñas sucias y se las meten a la boca".

- N5: "Cuando se comen alientos que tienen parásitos".

- N6: "Hay niños que comen muchos dulces, eso les causa parásitos, porque comen con las manos sucias".

- N7: "El banano, cuando a veces se daña y las personas se lo comen así".

- N8: "Por la tierra, jugando, por estar de pies descalzos".

Por otra parte, algunos padres de familia coinciden con los niños al manifestar que existe una correlación entre llevarse las manos sucias a la boca o tener contacto con objetos sucios, con las parasitosis intestinales:

- A2: "Debe ser porque uno a veces come con las manos sucias".

Se resalta que ninguno de los padres de familia menciona el hecho de que consumir agua contaminada constituye un factor de riesgo. Así mismo, algunos padres de familia manifiestan que los parásitos pueden ser 
adquiridos por cierto tipo de alimentos, aunque estén bien preparados y no estén en descomposición. Otros asocian el ciclo de transmisión de enfermedades virales -como la gripa- con las parasitosis intestinales, y la gran mayoría expresa que definitivamente no tienen conocimiento sobre el tema.

- A1: "Me han dicho que es el café con arroz, que es malo, que eso da mucho parásito, y los pone piponcitos".

- A6: "Yo me imagino que debe ser como cuando uno tiene gripa, porque yo digo, yo puedo ver a otro con gripa y yo me entro como a estornudar y me pone es mal, yo digo que será así".

Al indagar en los niños sobre lo que sucede cuando los parásitos se encuentran dentro del cuerpo de las personas, estos son conscientes que los parásitos pueden vivir, alimentarse y reproducirse dentro de sus organismos, y describen lo que para ellos hacen los parásitos dentro de las personas:

- N1: "Empiezan a comer. Se vuelven grandes".

- N4: "Algunos dicen que muerden los huesos"

- N5: "Quedan en la barriga y algunos se mueren".

- N8: "Se alimentan de la sangre".

- N11: "Se hartan de dulce, porque uno come".

- N12: "Hay algunos parásitos que se reproducen dentro de nuestro cuerpo y pueden morder nuestras partes".

Por su parte, los padres de familia manifiestan que los parásitos intestinales pueden desarrollarse dentro del cuerpo humano. Adicionalmente, algunos manifiestan que las fases de la luna pueden influenciar el desplazamiento de los parásitos hacía otras partes del cuerpo, y establecen conjeturas sobre el tipo de alientos que prefieren los parásitos, a partir de las preferencias alimenticias de los niños.

- A2: "Se alimentan de todo lo que uno consume, se alimentan de ellos adentro. Se quedan en el estómago, a veces unos se van pal cerebro, que es el que pone a los niños mal, más cuando hay la luna nueva. Se alimentan más del dulce, porque si al niño le gusta comer mucho dulce obvio que los parásitos..."

Cuando se pregunta a los niños por dónde salen los parásitos del cuerpo de las personas, y qué les pasa a estos patógenos cuando salen, los niños describen eventos o acciones asociadas a órganos específicos, por ejemplo, al manifestar que salen "por el popó" (por las heces fecales), está implícito que salen por el ano, mientras que al decir que salen "cuando vomitamos", la expulsión está asociada con la boca. Se resalta que todos los niños manifestaron que una vez los parásitos salen del cuerpo de las personas, estos mueren.

De igual forma, las respuestas de algunos padres de familia presentan concordancia con las de algunos niños al describir cómo salen los parásitos del cuerpo de las personas:

- A2: "Por el tantino, cuando hacen el popó".

- A3: "Por donde uno ensucia" (defeca)

- A4: "Hay niños que a veces lo botan por la boquita, por la naricita, por la caca".

Con respecto a lo que los padres de familia piensan que sucede una vez los parásitos salen del cuerpo de las personas, todos coinciden en que los parásitos mueren.

\section{Riesgos percibidos}

Al preguntarles a los niños si los parásitos intestinales que afectan a las personas pueden ser los mismos que afectan a los animales, la respuesta pone en evidencia que no se perciben riesgos ante el contacto con ellos. En relación con lo anterior, la respuesta predomínate en los menores fue la siguiente:

- N5: "Son otros parásitos".

Este tipo de respuestas también fue frecuente en el grupo focal de los padres; cuatro de ellos contestaron que los parásitos que afectan a los humanos son diferentes a los de los animales, cuatro contestaron que pueden ser los mismos, y dos manifestaron no tener conocimiento del tema:

- A4: "Los de los animales son otros. Animal es animal, persona es persona".

- A6: "No creo, porque los animales son 
más allegados al suelo que las personas".

\section{Tratamiento}

Cuando se indagó sobre el tratamiento que debe seguir una persona que tiene parásitos intestinales, los niños mencionan los medicamentos que consumen en la comunidad, incluyendo aquellos elaborados en la misma. Cabe destacar que los niños hacen referencia -principalmentea un tratamiento que es preparado con la planta conocida en la zona como Bajagua, y narran brevemente parte del procedimiento de elaboración.

- "Panela y hierbas. Si la persona mocha la planta para arriba y que vomita, y si la mocha para abajo hace popó".

- N3: "Mi mamá ponen a cocinar las hojas de salvia con la concha del mango y se lo dan a la persona, ella dice que se lo tiene que tomar todito, se tiene que tomar el agua, y la persona se va sintiendo mejor".

- N9: "Con purgante, hay uno que se Ilama albendazol".

Al indagar en los padres de familia sobre el tratamiento que deben recibir las personas parasitadas, seis de ellos coinciden en que se debe suministrar una infusión elaborada con la planta Bajagua, mezclada con panela. Según comentan los pobladores, el Bajagua tiene propiedades antiparasitarias y laxantes. En este sentido, al hacer referencia sobre este medicamento tradicional, las personas describen el ritual que se debe seguir para colectar la planta, la cantidad a utilizar, el proceso de preparación y la forma de administrarlo.
En virtud de lo anterior, quienes hablaron de esta planta, manifiestan que es imperativo que, al momento de colectar la planta, ésta debe ser cortada hacia abajo para que tenga un efecto purgante, de lo contrario -si es cortada hacía arriba- la persona que tome el medicamento puede vomitar los parásitos, lo que es considerado una práctica peligrosa (imagen 1). Se resalta además que tanto hombres como mujeres describen la forma en la que se debe preparar el medicamento, un hecho que Ilama la atención si se tiene en cuenta que los hombres manifestaron no tener conocimientos sobre los parásitos intestinales.

- A2: "El bajagua, eso es un palo que nace en la ciénaga, eso tiene que arrancarlo es pa' abajo, porque si lo arrancas pa' arriba vomitas. Estronchas pa' abajo 3 o 4 tallitos, la cocinas con la panela, y por aparte haces otro guarapo. Primero se va a tomar el purgante, y a la media hora te tomas el aguapanela".

- A9: "El bajagua, uno lo cocina con la panela y la deja en el sereno. Otro día compra uno la magnesia o sal de fruta le echa, ya uno se lo toma y ya". 


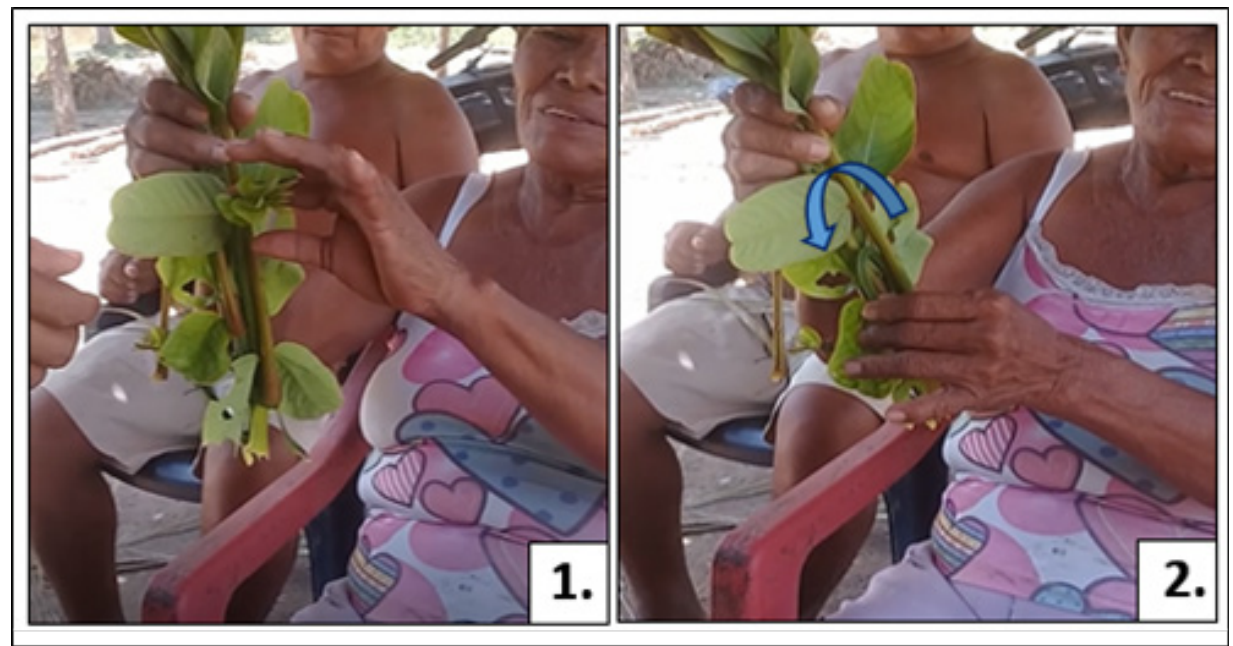

Imagen 1. Forma correcta de colectar la planta medicinal Bajagua.

Cabe resaltar varias personas manifestaron alguna variante en la preparación de la infusión de bajagua, al incluir elementos que son reconocidos por su efecto laxante, y que pueden actuar en sinergia con dicha planta, tales como sal de Epson (sulfato de magnesio), leche magnesia (hidróxido de magnesio) o piña (Ananas comosus (L.) Merr), y productos que mejoran la digestión; como la sal de frutas (bicarbonato de sodio) (21-24). De esta forma, se observan prácticas preventivas para afrontar diversos problemas de salud, que son producto de la mezcla del conocimiento tradicional con el saber biomédico.

\section{Prevención}

Al preguntar sobre cómo se pueden prevenir las parasitosis intestinales, los niños indican que no comer dulces y mantener la higiene es la mejor forma de prevenir los parásitos, tal como se aprecia a continuación:

$\begin{array}{ll}- & \text { N1: "Estar bien aseados". } \\ \text { - } & \text { N4: "Lavarse las manos". } \\ \text { - } & \text { N12: "No jugar con tierra". } \\ \text { ce". } & \end{array}$

Así mismo, una de las niñas entrevistadas comparte con los demás niños la forma en que ella previene las parasitosis intestinales, basada en su experiencia con esta enfermedad:

- $\quad$ N2: "a mí se me metió un parásito en la cabeza, el parásito del cerdo, cuando en mi casa criaban puercos, allá mi papá compraba los puerquitos chiquitos, entonces nosotros nos metíamos a jugar con ellos allí, y nosotros pasábamos pies descalzos, y cuando nos Ilevaron que nosotros convulsionamos, nos llevaron allá al hospital de San Andrés, allá nos transmitieron al hospital de Sincelejo, y allá nos dijeron que era el parásito del cerdo, había entrado en la cabeza, nos mandaron medicamentos, nos hospitalizaron, después de una año a mi hermano le dio, también convulsionó, y ya van tres veces que le pasa eso, y de allí fue que nos mandaron que estuviéramos aseados, cuando fuéramos a comer nos laváramos las manos, que pasáramos con chancletas".

Finalmente, al realizar esta pregunta en el grupo focal de los padres de familia, algunos manifiestan diversas formas de prevenir los parásitos, entre ellas mantener condiciones higiénicas saludables, el autocuidado; lavarse las manos, y el uso de purgantes. Algunos manifestaron no tener conocimiento sobre cómo prevenir las parasitosis.

- A1: "Lavarse las manos, recortarse las uñas... porque a veces tiene las uñas largas, juegan con tierra y las uñas quedan sucia... y allí mantienen parásitos".

- A2: "Con el aseo de uno".

- A3: "Yendo al baño y lavándose las manos, antes de comer lavándose las manos". 
- A4: "Cocinar los alimentos bien, lavarlos".

\section{DISCUSIÓN}

Los niños y las mujeres adultas que fueron sujeto de estudio poseen conocimientos sobre la causa-efecto de las parasitosis intestinales, en relación a la presentación de la enfermedad y a los efectos adversos que ésta ocasiona sobre la salud. No obstante, los niños relacionan el bruxismo (rechinar los dientes) con la presencia de parásitos, una creencia ampliamente diseminada en muchas comunidades, incluidas aquellas que no son indígenas. En este sentido, la asociación que se establece con el bruxismo se debe probablemente a las dinámicas de intercambio cultural que esta comunidad indígena tiene con otras no indígenas, en tanto la etiología del bruxismo es multifactorial y la relación con las parasitosis no ha sido concluyente $(17,25,26)$.

Al mencionar que se debe realizar búsqueda de parásitos intestinales en la zona perianal de los menores, se evidencia que existe un conocimiento que relaciona a las parasitosis intestinales con una parte específica del cuerpo o del sistema digestivo. Así mismo, se identifica la inspección corporal como un método diagnóstico de las enteroparasitosis en los niños, método que presenta concordancia con el conocimiento biomédico, en tanto algunas hembras de helmintos se desplazan hasta la zona perianal del menor afectado -principalmente en horario nocturno- para allí depositar sus huevos; momento en el que son visibles (27).

$\mathrm{Al}$ indagar sobre las vías que los parásitos intestinales emplean para entrar al cuerpo humano, la respuesta de los niños advierte que persisten percepciones tradicionales de riesgo mezcladas con algunas provenientes de la biomedicina. En este sentido, si bien la falta de aseo y caminar pies descalzos son factores de riesgo para desarrollar la enfermedad, comer dulces o frutas en descomposición no implica per se un riesgo para adquirir parásitos, de esta forma, al no tener claridad sobre cómo se adquieren, se aumenta el riesgo de infección.

Estudios similares señalan que los indígenas Mbya Guaraní de la provincia de Misiones (Ar- gentina), consideran -en al menos en dos de sus comunidades- que el desarrollo de los parásitos se encuentra asociado al consumo de carnes o dulces (28). Asimismo, la población Chinanteca, en México, considera que algunas fases de la luna pueden alterar el estado de reposo de "los bichos", lo que ocasiona trastornos gastrointestinales y diarreas (29), una postura similar a la observada en los indígenas Zenú que residen en la Vereda Berlín.

Con respecto a la identificación de algunos síntomas específicos de las parasitosis, los niños y adultos que fueron sujetos de estudio consideran a los parásitos como agentes que ocasionan desnutrición y anemia. De igual forma, en el pueblo indígena EmberaChamí de Colombia, se piensa que los parásitos consumen los alimentos de la persona que los hospeda, ocasionando disminución en las defensas, desequilibrio y finalmente la aparición de anemia (30). El sentido negativo que las comunidades le asignan a las parasitosis, puede ser considerado una ventaja por los profesionales de la salud, si se tiene en cuenta que algunas comunidades indígenas y no indígenas consideran que los parásitos intestinales son benéficos para la salud $(29,31)$.

Por otra parte, se identificó que los varones adultos que participaron en la presente investigación, desconocen cómo identificar los síntomas y las formas de prevención; un fenómeno que puede estar asociado a las prácticas culturales relacionadas con la distribución del trabajo en este tipo de comunidades (32). De esta manera, se identifica a la madre de familia o cuidadora, como un agente receptor de educación en cuanto a modos de prevención y tratamiento de la patología.

Al describir la forma, aspecto o color de los parásitos intestinales, los niños y los adultos coinciden en que estos tienen forma alargada, haciendo una descripción general de alguna especie de helminto. Sin embargo, los sujetos de estudio no realizan una representación verbal de los protozoarios. Lo anterior evidencia el desconocimiento que tienen acerca del tema, y los riesgos percibidos solo ante organismos que pueden ser observados a simple vista. En 
consecuencia, la falta de información sobre la morfología de los parásitos hace que las comunidades se enfoquen en la expulsión de las formas adultas de los helmintos.

Cuando los sujetos de estudio consideran que los parásitos intestinales mueren una vez salen del cuerpo de las personas, se encuentra concordancia con lo expresado por una comunidad de la zona rural del departamento de Cundinamarca, quienes consideran que las "lombrices" mueren cuando salen del organismo, una apreciación que juega un papel importante en el mantenimiento del ciclo de transmisión de los parásitos intestinales en ambas comunidades (19).

Con respecto al tratamiento para las parasitosis, en la comunidad residente en la vereda Berlín es ampliamente generalizado el uso de la planta Bajagua, mezclada en ocasiones con medicamentos que hacen parte de la medicina occidental. De esta forma, el empleo de este tipo de medicamentos evidencia el arraigo que estas prácticas tienen en dicha comunidad, en tanto la convivencia que tienen con la patología y los conocimientos que se han generado para contrarrestarlas, le ha conferido legitimidad.

\section{CONCLUSIONES}

Mujeres adultas y los niños que fueron sujeto de estudio, reconocen a los parásitos intestinales como causantes de padecimientos, e identifican -desde la óptica biomédica- algunos síntomas y formas de prevención relacionadas a esta patología, mezclada en ocasiones con el conocimiento tradicional. Así mismo, se identificó el desarrollo de acciones de autocuidado enfocadas al tratamiento de las parasitosis, las cuales se rigen por el conocimiento ancestral de los zenúes.

Tanto niños como adultos ignoran los riesgos que implica el consumo de agua contaminada o sin tratar. Así mismo, se desconocen los riesgos que pueden traer consigo el contacto con animales o con sus heces, lo que puede hacer que cualquier práctica de autocuidado resulte infructuosa al no tener las precauciones suficientes para interrumpir el ciclo de transmisión de las helmintiasis.

Un porcentaje importante de los hombres adultos que participaron en la investigación, carecen de conocimientos relacionados con la causa-efecto de la patología. En este sentido, se denota la distribución del trabajo, saberes y roles en la comunidad. En todos los casos, la información resulta relevante en tanto permiten adentrarse en la cultura de este pueblo para diseñar y desarrollar -a futuro- programas de prevención, promoción y educación en salud acordes al contexto.

Las condiciones ambientales y de saneamiento no deben ser consideradas exclusivamente como determinantes de la alta prevalencia de parasitosis intestinal, sino que debe abordarse dentro de un contexto sociocultural que puede ser potencialmente empleado para entender las condiciones en las que persisten estas infecciones. De esta manera, los conocimientos y prácticas de la comunidad de Berlín, permiten comprender la lógica que emplean estas personas para interactuar con los fenómenos salud-enfermedad, y proponer posibles programas en salud con enfoque diferencial, orientados a la prevención de la enfermedad y la promoción de la salud.

En este sentido, resulta imprescindible construir un programa de educación para la prevención de las parasitosis que sea producto de un diálogo de saberes y concertaciones bajo la lógica del respeto a la diferencia, que integre el saber tradicional con el biomédico, y que responda a la realidad en que vive este grupo social.

Se requieren investigaciones que permitan reconocer las nociones que las personas de esta comunidad tienen acerca de otras enfermedades transmisibles, a fin de identificar la causa de ciertas prácticas de riesgo que pueden estar relacionadas con las parasitosis. Se recomienda investigar, además, aquellos hábitos concretos que pueden disminuir la prevalencia de parásitos intestinales, como la forma en que se realiza el lavado de las manos o en la que se preparan los alimentos. 
Finalmente, se exhorta la implementación estudios enfocados en determinar la prevalencia parasitaria y el estado nutricional de los niños que residen en la comunidad indígena de Berlín, en tanto que una alta prevalencia de parásitos, aunado a deficiencias nutricionales y un sistema inmune poco desarrollado, podría potencializar un inadecuado desarrollo físico y cognitivo en los menores, con graves consecuencias para los mismos.

\section{Agradecimientos}

A la comunidad indígena Zenú residente en la vereda Berlín, al capitán menor del cabildo de Berlín, y a los docentes Julián Vanegas Barbosa, Lina Támara Osorio, Oscar Acosta Rojas y Marco Guevara Vega.

\section{Conflicto de intereses}

Los autores declaran no tener conflicto de intereses. Investigación financiada con recursos propios.

\section{REFERENCIAS}

1. Claros, A. M., Peña, M. M., López, M. G., Pérez, R. P., \& Fontelos PM. Parasitosis intestinales. Infectología pediátrica. 2012;7788.

2. McDonald $\mathrm{V}$. Parasites in the gastrointestinal tract. Parasite Immunol. 2003;25(5):231-4

3. World Health Organization. Prevention and Control of intestinal parasitic infections. Report of a WHO Expert committee. 2000. p. 88.

4. Carmona-Fonseca J. Malaria, desnutrición y parasitosis intestinal en los niños colombianos: Interrelaciones. latreia. 2004;17(4):354-69.

5. Díaz A I, Rivero R Z, Bracho M Á, Castellanos S M, Acurero E, Calchi L M, et al. Prevalencia de enteroparásitos en niños de la etnia Yukpa de Toromo, Estado Zulia, Venezuela. Rev Med Chil. 2006;134(1):72-8.

6. Nicholls S. Parasitismo intestinal y su relación con el saneamiento ambiental y las condiciones sociales en Latinoamérica y el Caribe. Biomédica. 2016;36(4):495-7.

7. Mehraj V, Hatcher J, Akhtar S, Rafique G, Beg MA. Prevalence and Factors Associated with Intestinal Parasitic Infection among Children in an Urban Slum of Karachi. 2008;3(11).

8. Hawdon JM. Controlling Soil-Transmitted Helminths : Time to Think Inside the Box? 2014;100(2):166-88.

9. Partida-rodr $\mathrm{O}$, Nieves-ram ME, Moran $\mathrm{P}$, Rojas L, Portillo T, Gonz E, et al. Human Intestinal Microbiota : Interaction Between Parasites and the Host Immune Response. Arch Med Res. 2017;48(8):690-700.

10. Nakada-Tsukui, K. N. Immune Response of Amebiasis and Immune Evasion by Entamoeba histolytica. Front Immunol. 2016;7:175.

11. Gamboa MI, Basualdo JA, Córdoba MA, Pezzani BC, Minvielle MC, Lahitte HB. Distribution of intestinal parasitoses in relation to environmental and sociocultural parameters in La Plata, Argentina. J Helminthol. 2003;77(1):15-20.

12. Khieu $V$, Odermatt $P$, Mel Y. Anémie dans une école du Cambodge rural: détection, prevalence et liens avec les parsitoses intestinales et la malnutrition. Bull Soc Pathol Exot. 2006;(1):115-8.

13. Gómez J, Botto C, Zent S, Marín A, Sánchez J, Noguera C. Influencia del tipo de vivienda y del tamaño de asentamiento de comunidades indígenas Piaroa en la transmisión de helmintos intestinales. Interciencia. 2004;29:389-95.

14. Osman M, Safadi D El, Cian A, Benamrouz $S$, Nourrisson C, Poirier P, et al. Prevalence and Risk Factors for Intestinal Protozoan Infections with Cryptosporidium , Giardia , Blastocystis and Dientamoeba among Schoolchildren in Tripoli, Lebanon. PLoS Negl Trop Dis. 2016;10(3).

15. Alto Comisionado de las Naciones Unidas para los Refugiados. El bienestar de los pueblos y comunidades indígenas. Los indicadores de bienestar. Available from: https://es.scribd.com/document/144281906/ EL-BIENESTAR-DE-LOS-PUEBLOS-Y-CO- 
MUNIDADES-INDIGENAS.

16. Organización Mundial de la Salud. La salud de los pueblos indígenas. Nota descriptiva N³26 octubre de 2007. 2007;13-4. Available from: http://www.who.int/gender-equity-rights/knowledge/factsheet-indigenous-healthn-nov2007-spa.pdf

17. Cabildo mayor regional del pueblo Zenú. Proyecto educativo comunitario "Sentir y pensar Zenú". CMRPZ., editor. San Andrés de Sotavento-Córdoba.; 2016. 160 p.

18. Secretaría de salud municipal de Tuchín. Análisis de situación de salud con el modelo de los determinantes sociales de salud del municipio de Tuchín-2016. [Internet]. 2016. Available from: http://tuchincordoba. micolombiadigital.gov.co/sites/tuchincordoba/content/files/000023/1112_actualizacion-asis-tuchin-2016--2017-final-1-corrgido.pdf.

19. Díaz $P$, Sc AM, Inés $L$, Álvarez $M$, Fernando D, Rosales C, et al. Conocimientos, actitudes y prácticas sobre las geohelmintiasis en una comunidad rural de Colombia. 2010;18(45):12-22.

20. Holdridge L. Ecología basada en zonas de vida. San José, Costa Rica: Editorial IICA: 1992.

21. Snyder, B. K., \& Clark RF. Effect of magnesium hydroxide administration on iron absorption after a supratherapeutic dose of ferrous sulfate in human volunteers: a randomized controlled trial. Ann Emerg Med. 1999;33(4):400-5.

22. Dupont C, Campagne A, Constant F. Efficacy and safety of a magnesium sulfaterich natural mineral water for patients with functional constipation. Clin Gastroenterol Hepatol. 2014;12(8):1280-7.

23. Fahim M, Shahdaat M, Sayeed B, Mia MMK. Ethnopharmacological survey of medicinal plants used by indigenous and tribal people in Rangamati, Bangladesh. J Ethnopharma- col. 2012;144(3):627-37.

24. Kumalasari, R., Herminiati, A., \& Andriansyah RCE. Pineapple Peel as a Potential Source of Dietary Fiber. In: Proceedings of international conference on appropriate technology development (ICATDev). 2015.

25. Berger M, Szalewski L, Szkutnik J, Ginszt M. Direct Different association between specific manifestations of bruxism and temporomandibular disorder pain. Neurol Neurochir Pol. 2017;51(1):7-11.

26. Kalamir A, Ã HC, Pollard H, Sc M, VitieIlo AL, Bonello R. TMD and the problem of bruxism . A review. J Bodyw Mov Ther. 2007;11(3):183-93.

27. Caumes, J. L., Chevalier, B., \& Klotz F. Oxiuros y oxiuriasis. EMC - Pediatría. 2002;37(2):1-5.

28. Murillo, M. D. P. D., Sánchez, N. A. R., \& García SDO. El sentido de las enfermedades por parásitos intestinales en poblaciones americanas, identificando dilemas bioéticos. Rev Latinoam Bioet. 2013;13(1):96111.

29. Sesia P. Los padecimientos gastrointestinales entre los chinantecos de Oaxaca: aspectos denotativos y connotativos del modelo etnomédico. Alteridades. 1999;9(17):7184.

30. Cardona J, Rivera Y. Representaciones sociales sobre medicina tradicional y enfermedades foraneas en indigenas EmberaChamí de Colombia. Rev Cuba Salud Publica. 2012;38(3):471-83.

31. Pebley A, Hurt E, Goldman N. Beliefs about children's illness among rural Guatemalan women. RAND CORP St MONICA CA. 1996;

32. Gaviria LM, Soscue D, Campo-Polanco LF, Cardona-Arias J, Galván-Díaz AL. Prevalencia de parasitosis intestinal, anemia y desnutrición en niños de un resguardo indígena Nasa, Cauca, Colombia, 2015. Rev Fac Nac Salud Pública. 2017;35(3):390-9. 